\title{
The Relaxed Universe: towards solving the cosmological constant problem dynamically from an effective action functional of gravity
}

\author{
Florian Bauer ${ }^{1}$, Joan Solà ${ }^{2}$ \\ HEP Group, Dept. ECM and Institut de Ciències del Cosmos \\ Univ. de Barcelona, Av. Diagonal 647, E-08028 Barcelona, Catalonia, Spain
}

Hrvoje Štefančid ${ }^{3}$

Theoretical Physics Division, Rudjer Bošković Institute

PO Box 180, HR-10002 Zagreb, Croatia

\begin{abstract}
We present an unconventional approach for addressing the old cosmological constant (CC) problem in a class of $F(R, \mathcal{G})$ models of modified gravity. For a $\mathrm{CC}$ of arbitrary size and sign the corresponding cosmological evolution follows an expansion history which strikingly resembles that of our real universe. The effects of the large $\mathrm{CC}$ are relaxed dynamically and there is no fine-tuning at any stage. In addition, the relaxation mechanism alleviates the coincidence problem. The upshot is that a large cosmological constant and the observed cosmic expansion history coexist peacefully in the Relaxed Universe. This model universe can be thought of as an interesting preliminary solution of the cosmological constant problem, in the sense that it provides a successful dynamical mechanism able to completely avoid the fine-tuning problem (the toughest aspect of the CC problem). However, since the Relaxed Universe is formulated within the context of modified gravity, it may still suffer of some of the known issues associated with these theories, and therefore it can be viewed only as a toy-model proposal towards a final solution of the CC problem.
\end{abstract}

\footnotetext{
${ }^{1}$ fbauer@ecm.ub.es

${ }^{2}$ sola@ecm.ub.es

${ }^{3}$ shrvoje@thphys.irb.hr
} 


\section{Introduction}

The triumph of modern physics is largely reflected in our ability to understand the properties of the universe and its dynamics from the fundamental physical theories. Yet there is a prediction of quantum field theory and string theory which is grossly inconsistent with the existence of the observed universe. The vacuum energy density calculated in these theories can be so large in absolute value $\left(\left|\rho_{\Lambda}\right| \simeq M_{X}^{4}\right.$, with $M_{X}$ around the Planck mass) that the universe would either recollapse immediately after the Big Bang or the formation of galaxies that we observe would be prevented. This huge gap in our understanding of nature, known as the old cosmological constant problem, is one of the most important unresolved issues in fundamental physics [1, 2]. The cosmological constant (CC) receives numerous contributions from zero point energies of quantum fields and phase transitions in the early universe, along with an arbitrary additive constant as allowed by general covariance. These effects are of very different orders of magnitude, and all of them are many orders of magnitude larger than the value consistent with the observations,

$\rho_{\Lambda}^{0} \approx\left(2.3 \times 10^{-3} \mathrm{eV}\right)^{4}$. There is the possibility that these disparate contributions might cancel to produce the observed value $\rho_{\Lambda}^{0}$, but this would entail an unacceptably large fine-tuning. Definitely, we have to find another, more natural, solution.

There have been numerous attempts to solve the old CC problem, e.g. a recent one is [3]. Many, if not all of them, and especially those based on dynamical scalar fields, still suffer from severe fine-tuning troubles in one form or another [1. Rather than replacing the CC by scalar fields, one may think of the CC term as an effective quantity $\rho_{\Lambda \text { eff }}$ evolving with the expansion rate of the universe, $H$. The approach to a dynamical CC in quantum field theory has been recently reemphasized in [4, and it has proven fruitful in tackling the cosmic coincidence problem [5]. Maybe it can also be useful to deal with the old CC problem. Thus, instead of looking for the solution in the matter sector, the possibility that it could have a gravitational origin should be seriously investigated. This might provide a well behaved "effective CC term" $\rho_{\Lambda \mathrm{eff}}(H)$, viz. one which is very large in the early universe $\left(H \simeq M_{X}\right)$ but very small at present $\left(H \simeq H_{0}\right)$. In this letter, we present action functionals of modified gravity which incorporate a very large $\left|\rho_{\Lambda}\right|\left(\gg \rho_{\Lambda}^{0}\right)$ and still result in a cosmological expansion with a measurable $\rho_{\Lambda \mathrm{eff}}(H)$ which is (very) close to the dynamics of the benchmark $\Lambda \mathrm{CDM}$ cosmological model. The striking feature of these modified gravity models is that they relax a large $\mathrm{CC}$ without fine-tuning. The gravitational mechanism for equilibrating the effects of a large $\mathrm{CC}$ we refer to as the relaxation mechanism whereas the cosmological models in which this mechanism is implemented we call the Relaxed Universe.

\section{Dynamical relaxation of the cosmological term}

An essential ingredient of the new universe is the feedback between the dynamics of space-time and the functioning of the relaxation mechanism. The very expansion owing to the existence of a large CC sets the relaxation mechanism in motion. The effects of modified gravity equilibrate (dynamically) the effects of the large CC. As a result, a realistic universe with a standard sequence of radiation dominated, matter dominated and accelerating epochs unfolds automatically. The 
relaxation mechanism based on the component with an inhomogeneous equation of state (EOS) was introduced in [6], while in [7] it was shown that the inhomogeneous EOS is an effective description of modified gravity effects. The following step was to construct a viable cosmological model.

In Ref. [8] we have proposed a scenario which achieves this goal in a dynamical way, thereby avoiding fine-tuning. In a flat FLRW cosmos, we introduced a dynamical vacuum component, which counter-acts the effect of a large $\left|\rho_{\Lambda}\right|$. The total vacuum energy density was found to be $\rho_{\Lambda \text { eff }}=\rho_{\Lambda}+\beta / B$, where $\beta$ is a constant parameter, and the function $B$ representing the heart of the relaxation mechanism will follow shortly. First, suppose that $\rho_{\Lambda} \sim-M_{\mathrm{EW}}^{4}$ (with $M_{\mathrm{EW}}=\mathcal{O}(100) \mathrm{GeV}$ ) as given by the large (and negative) vacuum contribution from the Higgs potential in the electro-weak phase transition. Without counter-measures, the negative $\rho_{\Lambda}$ would let the universe quickly collapse into a catastrophic Big Crunch. To prevent this, one could artificially add a fine-tuned CC counter-term. Our relaxation mechanism, though, will do the job automatically. Let us illustrate it first with a toy model in the radiation era, where the deceleration parameter $q=-\ddot{a} a / \dot{a}^{2}$ is close to but smaller than unity, $q \lesssim 1$. In this epoch, consider $\beta>0$ and $B=H^{2}(1-q)$ given in terms of $q$ and the Hubble rate $H=\dot{a} / a$. Clearly, the mechanism will stabilize the expansion, because the big $\rho_{\Lambda}<0$ wants to rapidly initiate the collapse, thereby pushing the deceleration $q$ closer to 1 . However, $q$ can never reach 1 because the second term $\beta /\left[H^{2}(1-q)\right]>0$ in $\rho_{\Lambda \text { eff }}$ will become sufficiently large to compensate the negative CC, finally resulting in $\left|\rho_{\Lambda \text { eff }}\right| \ll\left|\rho_{\Lambda}\right|$ - the CC has been relaxed. And vice versa: if $\beta / B>0$ would dominate over $\rho_{\Lambda}$, the value of $q$ would move away from $q \approx 1$ towards smaller values due to the large positive vacuum energy. Consequently, $\beta /\left[H^{2}(1-q)\right]$ will decrease and $q$ will reach a stable point where $\rho_{\Lambda}$ and $\beta / B$ compensate each other. The resulting universe will expand like a radiation cosmos since small changes of $q$ around $q \simeq 1$ are sufficient to keep both terms of $\rho_{\Lambda \text { eff }}$ in equilibrium.

\section{A more realistic model}

To apply the CC relaxation mechanism to the whole Big Bang expansion history, consider the more sophisticated B-function in $\rho_{\Lambda \text { eff }}$ above

$$
B=\frac{2}{3} R^{2}+\frac{1}{2} \mathcal{G}+(y R)^{3},
$$

involving only the Ricci scalar $R=6 H^{2}(1-q)$ and the Gauß-Bonnet term $\mathcal{G}=-24 H^{4} q$, both computed in the FLRW metric. Thus, $B$ reads explicitly

$$
B=24 H^{4}\left(q-\frac{1}{2}\right)(q-2)+H^{6}[6 y(1-q)]^{3} .
$$

It is easy to see that the second term, proportional to $H^{6}(1-q)^{3}$, will be responsible for the CC relaxation in the radiation regime $(q \simeq 1)$, where the Hubble rate was large. Since, however, $H$ decreases with time, the first term $\propto H^{4}(q-1 / 2)$, with a lower power of $H$, will eventually dominate and relax the $\mathrm{CC}$ in the matter era, where $q \simeq 1 / 2$. The arguments for the dynamical stabilization in $\rho_{\Lambda \text { eff }}$ are exactly the same as in the toy model above. Another novelty is the fixed 
parameter $y \sim H_{\mathrm{eq}}^{-2 / 3}$, which is related to the Hubble rate $H_{\mathrm{eq}} \sim 10^{5} H_{0}$ at the time of matterradiation equality, where $H_{0} \sim 10^{-42} \mathrm{GeV}$ is the current value. In the late accelerating universe, $q<0$ and the tiny expansion rate $H \approx H_{0}$ makes $B \sim H^{4}$ the relevant term. In summary, the smallness of the denominator $B$ in $\rho_{\Lambda \text { eff }}$ during all cosmological epochs is the clue to the relaxation mechanism. An additional physical argument in favor of it is the growth of inhomogeneities, which is in accord with observations [9].

This is a good place to demonstrate the absence of fine-tuning in our mechanism. Let us ignore $\mathcal{O}(1)$ factors in the Friedmann equation $\rho_{c}=\rho_{m}+\rho_{\Lambda}+\beta / H^{4}$ at late times. Since $\rho_{\Lambda}$ and the compensating term $\beta / H^{4}$ are much larger than the matter density $\rho_{m}$ and the critical energy density $\rho_{c}$, we find, in very good approximation, $H^{4}=\left|\beta / \rho_{\Lambda}\right|$ at late times. Obviously, small changes in the parameter $\beta$ will induce only small changes in the Hubble rate $H$. Hence, it suffices to fix the order of magnitude of $\beta$ to obtain a small $H \simeq H_{0}$. This has nothing to do with fine tuning. For instance, take a typical GUT value $\left|\rho_{\Lambda}\right| \simeq M_{X}^{4}$, with $M_{X}=10^{16} \mathrm{GeV}$; then $\beta=M^{8}$ with $M \sim 10^{-4} \mathrm{eV}$ (of order of a light neutrino mass) would do. An essential result thus follows: large $\left|\rho_{\Lambda}\right|$ guarantees small asymptotic $H \simeq H_{0}$ for reasonable $M$.

\section{An action functional formulation of the relaxation mechanism}

Next we implement the CC relaxation mechanism, here for the first time in the modified gravity setup and in the metric formalism [10, 11]. The crucial part of the model is a function $F(R, \mathcal{G})$ of the Ricci scalar $R$ and the Gauß-Bonnet invariant $\mathcal{G}$. The complete action functional of our cosmological model is the following:

$$
\mathcal{S}=\int d^{4} x \sqrt{|g|}\left[\frac{R}{16 \pi G_{N}}-\rho_{\Lambda}-\beta F(R, \mathcal{G})+\mathcal{L}_{\text {mat }}\right]
$$

where $\mathcal{L}_{\text {mat }}$ is the matter Lagrangian, and $\rho_{\Lambda}$ an arbitrarily large cosmological constant. $G_{N}$ is the Newton constant and the parameter $\beta$ will also be dimensionful in general. The variational principle $\delta \mathcal{S} / \delta g^{a b}=0$ then leads straightforwardly to the Einstein equations,

$$
G_{a b}=-8 \pi G_{N}\left[g_{a b} \rho_{\Lambda}+2 \beta E_{a b}+T_{a b}\right],
$$

involving the Einstein tensor $G_{a b}=R_{a b}-\frac{1}{2} g_{a b} R$ and the energy-momentum tensor $T_{a b}$ of matter. Additionally, there is a new tensor $E_{a b}$ coming solely from the $F(R, \mathcal{G})$ term in the action (3). On a spatially flat FLRW background with line element $d s^{2}=d t^{2}-a^{2}(t) d \vec{x}^{2}$ and scale factor $a(t)$, the tensor components in Eq. (4) are given by $G_{0}^{0}=-3 H^{2}, G^{i}{ }_{j}=-\delta^{i}{ }_{j}\left(2 \dot{H}+3 H^{2}\right)$ and

$$
\begin{aligned}
E_{0}^{0} & =\left[\frac{1}{2} F(R, \mathcal{G})-3\left(\dot{H}+H^{2}\right) F^{R}+3 H \dot{F}^{R}\right. \\
& \left.-12 H^{2}\left(\dot{H}+H^{2}\right) F^{\mathcal{G}}+12 H^{3} \dot{F}^{\mathcal{G}}\right], \\
E_{j}^{i} & =\delta^{i}{ }_{j}\left[\frac{1}{2} F(R, \mathcal{G})-\left(\dot{H}+3 H^{2}\right) F^{R}+2 H \dot{F}^{R}+\ddot{F}^{R}\right. \\
& \left.+\left(\dot{H}+H^{2}\right)\left(8 H \dot{F}^{\mathcal{G}}-12 H^{2} F^{\mathcal{G}}\right)+4 H^{2} \ddot{F}^{\mathcal{G}}\right],
\end{aligned}
$$


where $F^{R}$ and $F^{\mathcal{G}}$ are partial derivatives of $F$ with respect to $R$ and $\mathcal{G}$, respectively, and $\dot{H}=$ $-H^{2}(q+1)$.

The matter sector is described by the standard energy-momentum tensor of an ideal fluid, with proper energy density $\rho=T_{0}^{0}$ and pressure $p=-T_{i}^{i} / 3$ (summed repeated indices). Similarly, the $F(R, \mathcal{G})$-functional induces the effective energy density and pressure

$$
\rho_{F}=2 \beta E_{0}^{0} \text { and } p_{F}=-2 \beta E_{i}^{i} / 3 .
$$

Since $G_{a b}$ and $E_{a b}$ are covariantly conserved, $T_{a b}$ is conserved, too. Therefore, the Bianchi identity on the FLRW background, $\dot{\rho}+3 H(\rho+p)=0$, is valid for both matter $\left(\rho_{m}, p_{m}\right)$ and $F$-components $\left(\rho_{F}, p_{F}\right)$, and hence also for $\left(\rho_{\Lambda \text { eff }}, p_{\Lambda \text { eff }}\right)$, where $\rho_{\Lambda \text { eff }} \equiv \rho_{\Lambda}+\rho_{F}$ is the total energy density of the effective vacuum sector and $p_{\Lambda \text { eff }} \equiv-\rho_{\Lambda}+p_{F}$ denotes the corresponding pressure. The generalized Friedmann-Lemaître equations read:

$$
\begin{aligned}
3 H^{2} & =8 \pi G_{N}\left(\rho_{m}+\rho_{r}+\rho_{\Lambda \mathrm{eff}}\right), \\
H^{2}(q-1 / 2) & =4 \pi G_{N}\left(p_{r}+p_{\Lambda \mathrm{eff}}\right) .
\end{aligned}
$$

They contain the energy densities of pressureless matter $\rho_{m}=\rho_{m}^{0} a^{-3}$ and radiation $\rho_{r}=\rho_{r}^{0} a^{-4}$ with pressure $p_{r}=\rho_{r} / 3$, and the effective vacuum components.

Motivated by the toy-model discussion above, the function $F$ in our setup is taken in the simple form $F(R, \mathcal{G})=1 / B$, with $B$ given in Eq. (1). The key to the relaxation mechanism in this functional framework is the observation that every derivative of $F$ in the expressions (5) and (6) yields another factor $B^{-1}$. Thus the induced quantities $\rho_{F}$ and $p_{F}$ adopt, in general, the structure

$$
\rho_{F}=\frac{N_{1}(H, q, \dot{q})}{B^{3}}, \quad p_{F}=\frac{N_{2}(H, q, \dot{q}, \ddot{q})}{B^{4}},
$$

where $N_{1,2}$ are functions not proportional to $B$. Correspondingly, the effective vacuum energy density adopts the form $\rho_{\Lambda \text { eff }}=\rho_{\Lambda}+N_{1} / B^{3}$ and, in complete analogy with the toy-model discussion, it can be small because the large $\rho_{\Lambda}$ can be dynamically compensated by letting $B$ become sufficiently small (but non-zero), i.e. $B \rightarrow 0$ - hereafter referred to as "relaxation condition".

Note that, at the equilibrium point (e.g. $q \simeq 1$ in the radiation era), both terms in $\rho_{\Lambda \text { eff }}$ are almost equal to each other, apart from opposite signs. Therefore, $\rho_{F}$ behaves approximately as another (large) cosmological constant. Obviously, $\rho_{\Lambda \text { eff }}$ is not constant in general, because $\rho_{F}=\rho_{F}(H, q, \dot{q})$ is time-dependent. In addition, there is a corresponding compensation of the pressure terms $p_{F}$ and $-\rho_{\Lambda}$ in $p_{\Lambda \text { eff. }}$. Finally, since the functions (5) and (6) differ from each other, the effective EOS, $\omega=p_{\Lambda \text { eff }} / \rho_{\Lambda \text { eff }}$, will be in general a non-trivial function of time or redshift. This should have phenomenological implications.

\section{Analytical and numerical analysis of the model}

In the following, we will describe approximately the cosmic evolution by making use of the $\mathrm{CC}$ relaxation condition, which is equivalent to $\left|\rho_{\Lambda \text { eff }}\right| \ll\left|\rho_{\Lambda}\right|$. Remember that $B$ never vanishes. Our analysis will be supported by exact numerical results (cf. Fig. 1) obtained by solving directly the Friedmann equation (8) with $\rho_{F}$ given in Eq. (7). 
Let us start in the matter era where $q \approx 1 / 2$ and $H^{2} \sim \rho_{m} \propto a^{-3}$. Applying the relaxation condition in Eq. (2) leads to $(q-1 / 2) \propto H^{2}$, and Eq. (9) renders

$$
H^{2}(q-1 / 2)=4 \pi G_{N}\left(p_{r}+p_{\Lambda \mathrm{eff}}\right) \propto H^{4} \propto a^{-6},
$$

whereupon $p_{\Lambda \text { eff }}=-\rho_{r} / 3+c_{1} a^{-6}$, with $c_{1}$ a constant; and then via the Bianchi identity for $\left(\rho_{\Lambda \text { eff }}, p_{\Lambda \text { eff }}\right)$ we find $\rho_{\Lambda \text { eff }}=c_{2} a^{-3}-\rho_{r}+c_{1} a^{-6}$, the effective vacuum energy density, with $c_{2}$ another constant. Thence

$$
\omega=\frac{p_{\Lambda \mathrm{eff}}}{\rho_{\Lambda \mathrm{eff}}}=\frac{-\rho_{r} / 3+c_{1} a^{-6}}{c_{2} a^{-3}-\rho_{r}+c_{1} a^{-6}}
$$

is the corresponding EOS, which interpolates between dust matter $(\omega \rightarrow 0)$ at late times and radiation $(\omega \rightarrow 1 / 3)$ in the early matter era. Depending on the integration constant $c_{2}$ a pole might occur in $\omega$ when $\rho_{\Lambda \text { eff }}$ changes its sign, see Fig. 1. It is worth noticing that $\rho_{\Lambda \text { eff }}$ behaves like dark matter in this epoch, and we could speculate on incorporating both dark matter and dark energy into $\rho_{\Lambda \text { eff }}$. Apart from that, we remark the approximate tracking relation $\rho_{\Lambda \text { eff }} \propto \rho_{m}$, which can be considered a cornerstone for solving the coincidence problem.

We expect that the relaxation mechanism became active only as of the early radiation era, because $F=1 / B \sim H^{-6}$ in Eq. (3) was sub-dominant during the preceding periods of inflation and reheating, where $q$ was far from 1 . However, deep in the radiation epoch, before the matter era, we find $q \approx 1$ and $F$ becomes very important. Then $H^{2} \sim \rho_{r} \propto a^{-4}$ implies $R=6 H^{2}(1-q) \propto$ $H^{\frac{4}{3}} \propto a^{-\frac{8}{3}}$ upon using the relaxation condition in Eq. (2). Moreover, Eqs. (8) and (9) lead to the relation

$$
3 H^{2}(q-1)=4 \pi G_{N}\left[3 p_{\Lambda \mathrm{eff}}-\left(\rho_{\Lambda \mathrm{eff}}+\rho_{m}\right)\right] \propto a^{-8 / 3},
$$

and thus to $p_{\Lambda \text { eff }}=\left(\rho_{m}+\rho_{\Lambda \text { eff }}\right) / 3+c_{3} a^{-8 / 3}$, for constant $c_{3}$. From here the Bianchi identity determines $\rho_{\Lambda \text { eff }}=c_{4} a^{-4}-\rho_{m}-(9 / 4) c_{3} a^{-8 / 3}$, with a new a constant $c_{4}$. Accordingly, the EOS interpolates between radiation at early times and dust matter at later times:

$$
\omega=\frac{p_{\Lambda \mathrm{eff}}}{\rho_{\Lambda \mathrm{eff}}}=\frac{1}{3} \frac{1+\left(3 c_{3} / 4 c_{4}\right) a^{4 / 3}}{1-\left(\rho_{m}^{0} / c_{4}\right) a-\left(9 c_{3} / 4 c_{4}\right) a^{4 / 3}} .
$$

Again a pole in $\omega$ (visible in Fig. 1) can appear depending on $c_{4}$. As in the case of the matter epoch, it entails no physical singularity since all the energy densities remain finite; it merely reflects the change of sign of $\rho_{\Lambda \text { eff. }}$ Remarkably enough, we encounter the tracking property $\rho_{\Lambda \text { eff }} \propto \rho_{r} \propto a^{-4}$ also in the radiation era.

At late times, the universe leaves the matter epoch $\left(\rho_{m} \sim 1 / a^{3} \rightarrow 0\right)$ and gradually enters the "dark energy (DE) era". As $q$ departs now significantly from $1 / 2$ or 1 , the last dynamical resource left to the relaxation mechanism for compensating the big $\rho_{\Lambda}$, is to choose a very low value of $H$, whereby the DE epoch takes over somewhere near our time (cf. Fig. 1) - "cosmic coincidence". As the lowest power of $H$ becomes dominant in Eq. (2), we have $B \simeq 24 H^{4}(q-1 / 2)(q-2)$ and the full expression for $\rho_{F}$ in this regime can be computed in closed form:

$$
\frac{\rho_{F}}{\beta}=\frac{5 q^{2}-3 q-5}{2 H^{4}\left(2 q^{2}-5 q+2\right)^{2}}-\frac{\dot{q}\left(4 q^{2}-10 q+7\right)}{2 H^{5}\left(2 q^{2}-5 q+2\right)^{3}} .
$$




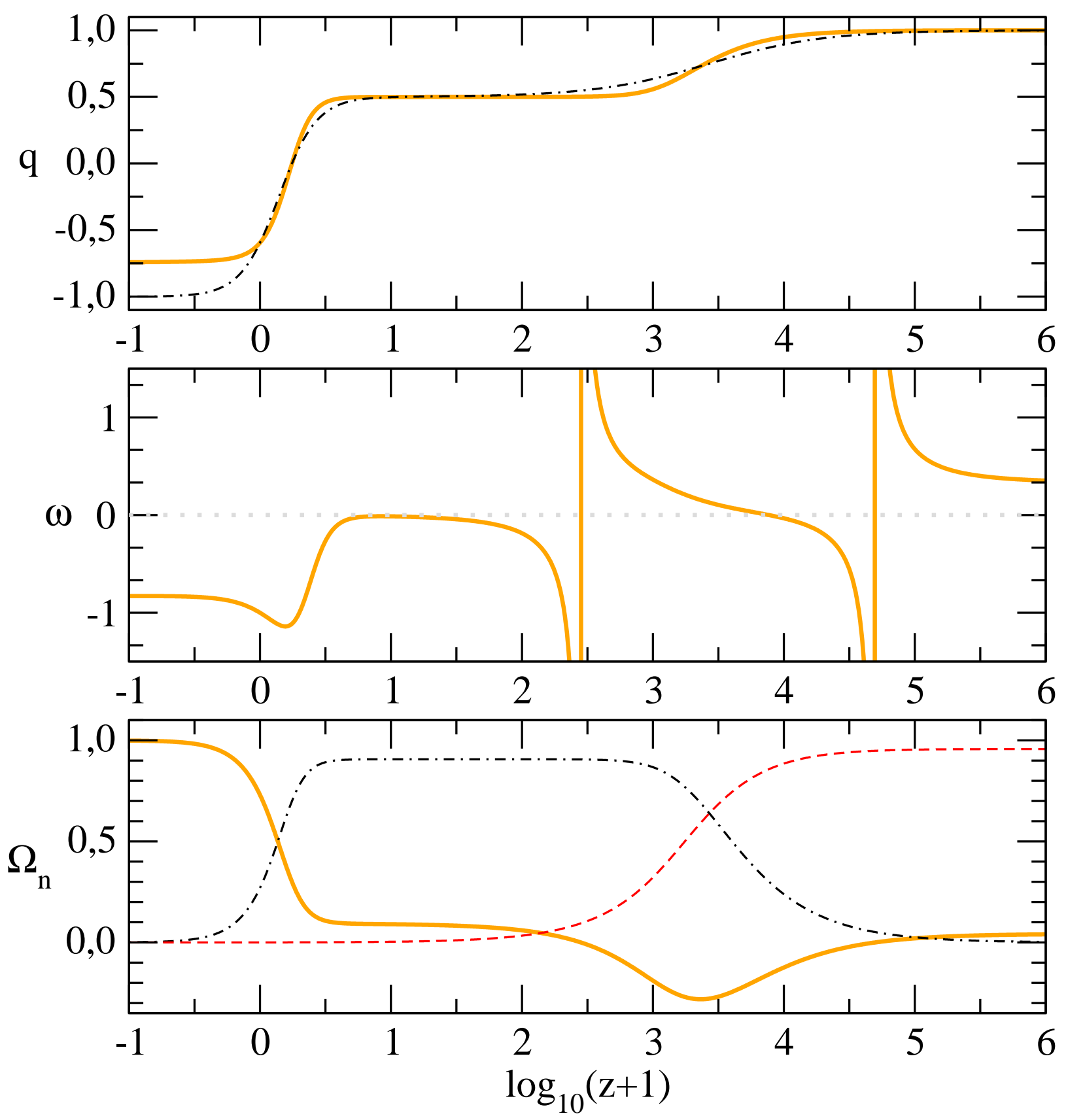

Figure 1: Deceleration parameter $q$, effective EOS $\omega=p_{\Lambda \text { eff }} / \rho_{\Lambda \text { eff }}$, and relative energy densities $\Omega_{n}=\rho_{n} / \rho_{c}$ of the effective vacuum $\rho_{\Lambda \text { eff }}$ (orange thick curve), matter $\rho_{m}$ (black dasheddotted) and radiation $\rho_{r}$ (red dashed) as functions of the redshift $z$. The thick orange curve in the $q$ plot corresponds to the relaxation model, and the black dashed-dotted curve to $\Lambda$ CDM. Model $F=1 / B$, with inputs $y=7 \times 10^{-4} H_{0}^{-2 / 3}, \rho_{\Lambda}=-10^{60} \rho_{c}^{0}, \Omega_{m}^{0}=0.27, \Omega_{r}^{0}=10^{-4}, q_{0} \approx-0.6$, $\dot{q}_{0}=-0.5 H_{0}$, with $\rho_{c}^{0}=3 H_{0}^{2} /\left(8 \pi G_{N}\right)$. 
This equation holds for the recent, current and asymptotic future times, i.e. whenever the universe is DE or matter dominated. With $H \rightarrow 0$, the previous expression would grow without limit, unless the numerators eventually tend to zero, too. Thus, the accelerating asymptotic solution ensues for $\dot{q} \rightarrow 0$ and $q \rightarrow(3-\sqrt{109}) / 10 \approx-0.74$, see Fig. 1. There are actually three solutions for $t \rightarrow \infty:$ a phantom phase, a de Sitter phase with constant $H$ and an asymptotic power-law expansion $a(t) \propto t^{r}$ with $r>1$. The previously found solution is of this sort, with $r \rightarrow 1 /(q+1) \approx 3.91$.

It is not difficult to work out generalized relaxation models, e.g. of the form $F=R^{n} / B^{m}$ with $m, n>0$. The crucial ingredient is the function $B$ in the denominator of $F$. Generally, this yields induced terms $\rho_{F}, p_{F} \propto B^{-s}$ with $s>0$ as a result of Eqs. (5 6), where derivatives of $F$ introduce more factors of $B$ in the denominators. One can readily show that the $\mathrm{CC}$ relaxation and tracking properties in the matter and radiation eras follow again from the relaxation condition. Furthermore, the parameter $\beta$ is related to a power of a mass scale $M$ as $|\beta|=M^{4-2 n+4 m}$. The size of $M$ can be estimated by applying the approximations $H, \dot{q} \sim H_{0}, q \in[-1,0]$, together with the condition $\left|\rho_{\Lambda}\right| \simeq\left|\rho_{F}\right| \simeq|\beta F|$, at $H \sim H_{0}$. Again we take $\left|\rho_{\Lambda}\right| \sim M_{X}^{4}$ for the standard GUT energy density $\left(M_{X}=10^{16} \mathrm{GeV}\right)$. In the case $(n, m)=(0,1)$, we recover the previous result $M^{8}=|\beta|=\mathcal{O}\left(\rho_{\Lambda} H_{0}^{4}\right)$, i.e. $M \sim 10^{-4} \mathrm{eV}$. Similarly, we obtain $M \sim 100 \mathrm{MeV} \sim \Lambda_{\mathrm{QCD}}$, for $(n, m)=(3,2)$; and $M \sim M_{X}$, for $(n, m)=(2,1)$. Remarkably, the mass parameter $M$ of the relaxation mechanism is not only completely free from fine-tuning problems, it also lies in a perfectly reasonable range of particle physics masses, possibly related to neutrinos, QCD or even GUT models.

\section{Conclusions}

To summarize, in this letter, we have unveiled a whole class of modified gravity action models which, despite holding an arbitrarily large CC at all times since the early epochs, display a very small "effective CC" at present, and without ever needing fine tuning. Like many modified gravity models our approach is not complete because of the existence of extra degrees of freedom and related problems, which require further considerations, see e.g. [11, 12]. Nevertheless, the Relaxed Universe scenario is not just another example for inducing late time cosmic acceleration, for the gravity modifications needed to dynamically compensate the large $\mathrm{CC}$ are crucial during the entire cosmic history. The mechanism also predicts remarkable tracking properties, and characteristic dynamical features in our recent past, which alleviate the coincidence problem. A more detailed account of this framework will be presented elsewhere.

Acknowledgments The authors have been supported by DIUE/CUR Generalitat de Catalunya under project 2009SGR502; FB and JS also by MEC and FEDER under project FPA2007-66665 and by the Consolider-Ingenio 2010 program CPAN CSD2007-00042, and HS also by the Ministry of Education, Science and Sports of the Republic of Croatia under contract No. 098-0982930-2864. 


\section{References}

[1] S. Weinberg, Rev. Mod. Phys. 61 (1989) 1.

[2] P.J.E. Peebles and B. Ratra, Rev. Mod. Phys. 75 (2003) 559; T. Padmanabhan, Phys. Rept. 380 (2003) 235; V. Sahni, A. Starobinsky, Int. J. Mod. Phys. A9 (2000) 373; S.M. Carroll, Living Rev. Rel. 4 (2001) 1; E.J. Copeland, M. Sami, S. Tsujikawa, Int. J. Mod. Phys. D15 (2006) 1753, and references therein.

[3] D. A. Demir, Found. Phys. 39 (2009) 1407.

[4] I. L. Shapiro, J. Solà, Phys. Lett. B682 (2009) 105.

[5] J. Grande, J. Solà, H. Štefančić, JCAP 0608:011 (2006).

[6] H. Štefančić, Phys. Lett. B670 (2009) 246.

[7] S. Nojiri, S. D. Odintsov, Phys. Rev. D 72 (2005) 023003.

[8] F. Bauer, J. Solà, H. Štefančić, Phys. Lett. B 678 (2009) 427, arXiv:0902.2215.

[9] F. Bauer, Class. Quant. Grav. 27 (2010) 055001, arXiv:0909.2237.

[10] S. Nojiri and S.D. Odintsov, Int. J. Geom. Meth. Mod. Phys. 4 (2007) 115; T.P. Sotiriou, V. Faraoni, arXiv:0805.1726 [gr-qc]; R. Woodard, Lect. Notes Phys. 720 (2007) 403.

[11] S.M. Carroll, V. Duvvuri, M. Trodden, M.S. Turner, Phys. Rev. D70 (2004) 043528; S. Nojiri, S.D. Odintsov, Phys. Rev. D68 (2003) 123512; S.M. Carroll, A. de Felice, V. Duvvuri, D.A. Easson, M. Trodden, M.S. Turner, Phys. Rev. D71 (2005) 063513.

[12] A. Hindawi, B.A. Ovrut, D. Waldram, Phys. Rev. D53 (1996) 5597; A. De Felice, M. Hindmarsh, M. Trodden, JCAP 0608 (2006) 005. 